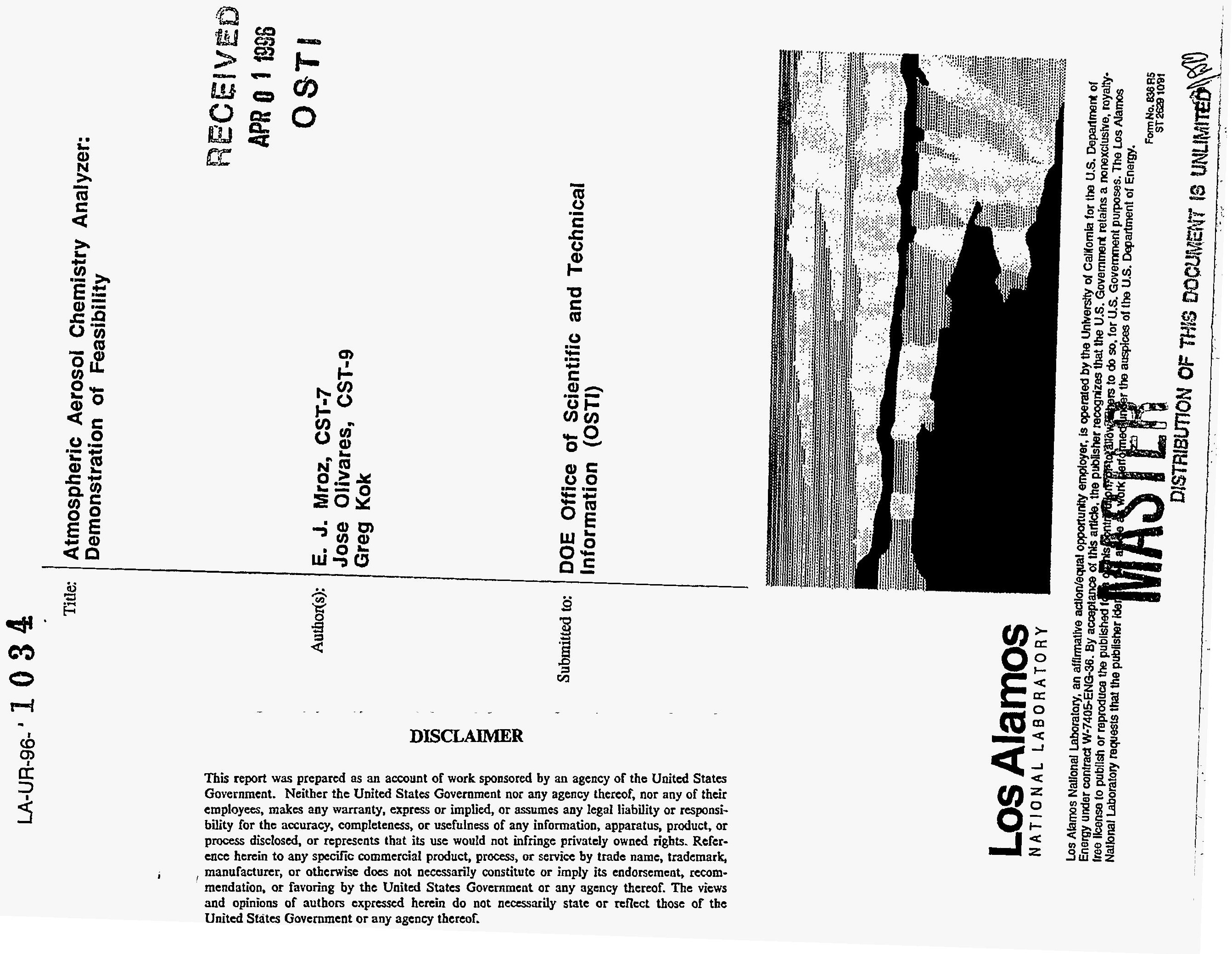




\section{DISCLAIMER}

Portions of this document may be illegible in electronic image products. Images are produced from the best available original document. 


\title{
Atmospheric Aerosol Chemistry Analyzer: Demonstration of Feasibility
}

\author{
E. J. Mroz*, Jose Olivares, and Greg Kok
}

\begin{abstract}
This is the final report of a three-year, Laboratory-Directed Research and Development (LDRD) project at the Los Alamos National Laboratory (LANL). The project objective was to demonstrate the technical feasibility of an Atmospheric Aerosol Chemistry Analyzer (AACA) that will provide a continuous, real-time analysis of the elemental (major, minor and trace) composition of atmospheric aerosols. The AACA concept is based on sampling the atmospheric aerosol through a wet cyclone scrubber that produces an aqueous suspension of the particles. This suspension can then be analyzed for elemental composition by ICP/MS or collected for subsequent analysis by other methods. The key technical challenge was to develop a wet cyclone aerosol sampler suitable for respirable particles found in ambient aerosols. We adapted an ultrasonic nebulizer to a conventional, commercially available, cyclone aerosol sampler and completed collection efficiency tests for the unit, which was shown to efficiently collect particles as small as 0.2 microns. We have completed the necessary basic research and have demonstrated the feasibility of the AACA concept.
\end{abstract}

\section{Background and Research Objectives}

Our ultimate goal was to develop a robust, transportable Atmospheric Aerosol Chemistry Analyzer (AACA) that will provide real-time analysis of the chemical composition of atmospheric aerosols. The AACA concept is illustrated in Figure 1 using a hypothetical hazardous waste incinerator as one example of several different areas where this technology might be applied. This example illustrates how the AACA concept could be employed in the stack or downwind of a hazardous waste incinerator. An operator in the plant could be sitting at a console monitoring a computer display of the periodic table of elements. If the ambient or

*Principal investigator, e-mail: mroz@lanl.gov 
stack concentration of hazardous element exceeded some threshold value, then the operator would be alerted and the operator could take appropriate action. Currently, there is no analytical methodology for achieving this kind of real-time chemical analysis of airborne particles.

The traditional method of aerosol analysis has always been a two-step process: filtration of the particles out of the air followed by chemical analysis of the filter in the laboratory by one or more analytical methods. But all methods are constrained by the trace element content of the filter material. The amount of particulate matter that must be collected on the filter to overcome the filter blank heavily influences the selection of sampling interval and flow rate. The need to bring the filter back to the laboratory for analysis has precluded achieving real-time or even near-real-time results in the field.

The purpose of this research was develop, test and demonstrate the aerosol sampling apparatus that is central to ensuring the feasibility of the AACA concept.

\section{Importance to LANL's Science and Technology Base and National R\&D Needs}

The AACA could contribute to several different technical areas that are important to LANL and national R\&D needs. These include environmental restoration and monitoring, continuous emissions monitoring, industrial hygiene, regional air pollution and source attribution, and chemical, biological and nuclear weapons non-proliferation and forensics.

Environmental Restoration and Monitoring. Fugitive dust emissions carrying hazardous materials are a significant problem for many environmental restoration projects. The AACA would be an in situ, real-time sensor of the elemental composition of these emissions. The AACA could be used to monitor and assess risk to site workers and the public from winddriven resuspension of contaminated soils around nuclear weapons complex facilities. As described below, we demonstrated the feasibility of the AACA concept by measuring the amount of uranium in fugitive dust produced by wind erosion of a uranium-contaminated firing site at Los Alamos.

Continuous Emissions Monitoring. The AACA could also serve as a real-time multi-element stack effluent monitor. This would be particularly valuable for processes such as incineration where the chemistry of the aerosol emissions changes rapidly depending on the chemistry of the waste materials passing through the incinerator (Fig. 1).

Industrial Hygiene. Industrial hygienists in a workplace containing potentially toxic amounts of aerosols need an aerosol sampling and analysis methodology that is specific and rapid. The present approach of filter sampling followed by chemical analysis does not yield the 
required results in a timely manner. The exposure risk to workers must be assessed continuously and in real time. The AACA can provide information on the airborne concentration of hazardous chemicals in near real time.

Regional Air Pollution and Source Attribution. Los Alamos scientists participated in an experiment to examine the impact of the coal-fired Navajo Generating Station in Page, Arizona, on the visibility at Grand Canyon and several other National Parks in the southwest US. Determining the trace element composition of the aerosol was an important component of that study because it permitted the identification of the sources contributing to the ambient aerosol. The existence of an AACA would permit measurements with the temporal resolution necessary to improve our understanding of the relationships among aerosol sources, atmospheric visibility and atmospheric transport.

\section{Chemical, Biological and Nuclear Weapons Non-proliferation and}

Forensics. The AACA technology is well-suited for rapid detection and identification of aerosols resulting from the development, testing and battle-field use of chemical, biological and nuclear weapons. Aerosols containing uranium, plutonium, their fission products and other elements are inevitably produced by the processing, enriching and/or machining of parts for nuclear weapons. Similarly, airborne chemical and biological warfare agents could be detected. Other possible applications include forensic use as a "sniffer" for aerosolized highexplosives, illegal drugs or other contraband materials.

\section{Scientific Approach and Results}

The key technical development necessary to demonstrate the feasibility of the AACA concept was to develop a method of efficiently transferring airborne particles as small as 0.2 microns diameter into an aqueous medium. That achieved, the aqueous suspension can then be analyzed by ICP/MS for most of the elements in the periodic table or by other analytical methods as may be appropriate for other airborne chemicals. We accomplished this by adapting the concept of a wet cyclone scrubber that is commonly used as an industrial air pollution control device and scaling it down to a transportable size and coupling it to whatever analytical device is appropriate for the aerosol being sampled. We call the sampler the Wet Cyclone Aerosol Sampler (WCAS).

Refer to Figure 2 for a sketch of the operational concept of the WCAS. In operation, an ultrasonic nebulizer continuously sprays a fine mist of water droplets (about 18 microns diameter) into a cyclone sampler at a rate of about $1 \mathrm{ml} / \mathrm{min}$. The water droplets are larger than the cutoff diameter of the cyclone and are driven to the walls of the cyclone by the centrifugal flow within the cyclone where they gather and drain down to a collection reservoir. 
Atmospheric particles entering the cyclone sampler are entrained in the vortex and are also transported toward the walls of the cyclone by centrifugal forces established by the cyclone geometry and the air flow rate. Particles that are larger than the cut-off diameter of the cyclone may impact the walls or may be captured by a water droplet and be subsequently washed down into a collection reservoir at the bottom of the cyclone. The aqueous suspension of atmospheric particles in the collection reservoir is continuously pumped out of the WCAS via a peristaltic pump. This suspension can go directly to either an analytical device such as an ICP/MS for analysis or, if immediate analysis is not feasible, the suspension can be collected by a conventional liquid fraction collector. These time-resolved samples can then be held for subsequent analysis by a suitable analytical method.

We conducted a series of experiments to assess the collection efficiency of the WCAS for four particle sizes $(0.83,0.40,0.25$ and 0.10 microns) at two air flowrates (28 and 40 $1 / \mathrm{min}$ ) and three nebulizer flowrates $(0.5,1.0$ and $1.5 \mathrm{ml} / \mathrm{min})$. Figure 3 shows the collection efficiency as a function of particle size for each of the three nebulizer flowrates at air flowrates of $28 \mathrm{l} / \mathrm{min}$ (Fig. 3a) and $40 \mathrm{l} / \mathrm{min}$ (Fig 3b). At 0.4 microns, the WCAS is $100 \%$ efficient even at $28 \mathrm{l} / \mathrm{min}$. This demonstrates that the addition of the water spray significantly enhances the performance of the cyclone. For all air flow and nebulizer conditions, the D50 is stable and between about 0.1 and 0.2 micron.

Measurements of Airborne $\mathrm{Be}, \mathrm{Pb}$ and $\mathrm{U}$. E-F Site is an abandoned high explosives firing site at Los Alamos National Laboratory. The soil is contaminated with $\mathrm{Be}$, $\mathrm{Pb}$ and $\mathrm{U}$. Soil uranium concentrations in excess of $400 \mathrm{ppm}$ are common. Our goal was to sample fugitive dust from drilling activity on the highly contaminated dirt mounds that marked the firing point of E-F Site.

We sampled the air downwind of the drilling activity on three days: 28 October, 1 November and 2 November, 1994. The sampling equipment was operated from the back of a pickup truck. The equipment was powered by a small $(3 \mathrm{KW})$ gasolinepowered electrical generator. This arrangement permitted mobility and allowed us to position the truck as necessary so that it was always downwind of the drilling activity. Analytical blanks were generated by diverting the air flow through an absolute filter before entering the wet cyclone scrubber. The sampling protocol was to collect an analytical blank for 15 minutes, followed by three 15 -minute samples and repeating this sequence throughout the experimental period.

During the experimental period we also measured the size distribution of the ambient aerosol using a PMS Lasair 310 optical particle counter. Data was acquired on a 15 minute time average to coincide with the time base of samples produced by the wet cyclone scrubber. 
Meteorological data was obtained from towers located within about two miles of E-F Site. The meteorological data is available on the same 15-minute time base as the sampling protocol.

The data from one of the towers was used to calculate a parameter known as the friction velocity, which is derived from the gradient in wind speed as a function of height. The friction velocity is a measure of the amount of stress applied to the surface by the wind. Resuspension of dust from soils has been shown to be related to the friction velocity. Differing soil types exhibit differing threshold friction velocities-the value above which resuspension of dust begins. For soils such as those at E-F site, the threshold friction velocity is estimated to be about $70 \mathrm{~cm} / \mathrm{s}$.

The samples were submitted for ICP/MS analysis for $\mathrm{Be}, \mathrm{Pb}$ and $\mathrm{U}$. Based on the statistics of the results of the analytical blanks we estimate our detection limits (1 sigma) for $\mathrm{Be}, \mathrm{Pb}$ and $\mathrm{U}$ to be $0.5,7$, and $0.2 \mathrm{ng} / \mathrm{m}^{3}$, respectively.

We present the results from 2 November because this day was characterized by persistent moderately strong S/SW winds throughout the day. The average wind speed was $4.4 \mathrm{~m} / \mathrm{s}$ and, more importantly, the mean friction velocity was $76 \pm 15 \mathrm{~cm} / \mathrm{s}$ with a maximum $100 \mathrm{~cm} / \mathrm{s}$ for one 15 -minute interval. During this period we sampled in a location that is about $900 \mathrm{ft}$ from the drilling activity. For Be we observed four positive values, ranging from 0.9 to $2.1 \mathrm{ng} / \mathrm{m}^{3}$. For $\mathrm{Pb}$ there as only one elevated period at $24 \mathrm{ng} / \mathrm{m}^{3}$. We observed a period of elevated uranium concentrations starting at 10:15 AM continuing until 3:45 PM. The range of the 19 observed values was from 0.3 to $3.2 \mathrm{ng} / \mathrm{m}^{3}$. The mean was $0.9 \pm 0.5 \mathrm{ng} / \mathrm{m}^{3}$. The median value was $0.7 \mathrm{ng} / \mathrm{m}^{3}$. We examined the set of the 19 data periods and found a positive correlation $(\mathrm{R} 2 \leq 0.5)$ among friction velocity, airborne uranium and the size distribution of the aerosol.

Figure 4 is a plot of the observed airborne uranium concentration vs. the friction velocity. A step-like increase in airborne uranium at friction velocity values above about $70 \mathrm{~cm} / \mathrm{s}$ is suggestive of the onset of resuspension. However, the contribution to airborne $U$ from drilling activities disturbing the soil surface may be part of the measured signal as well.

Conclusion. With LDRD support, we have successfully developed and tested a novel aerosol sampling apparatus that is central to the implementation of the AACA concept. We demonstrated that the WCAS efficiently collects airborne particles as small as 0.2 microns. We demonstrated detection limits for $\mathrm{Be}, \mathrm{Pb}$ and $\mathrm{U}$ of less than $1 \mathrm{ng} / \mathrm{m}^{3}$. 
Figure 1

ReatTime Elemental Analysis of Atmospheric Aerosols

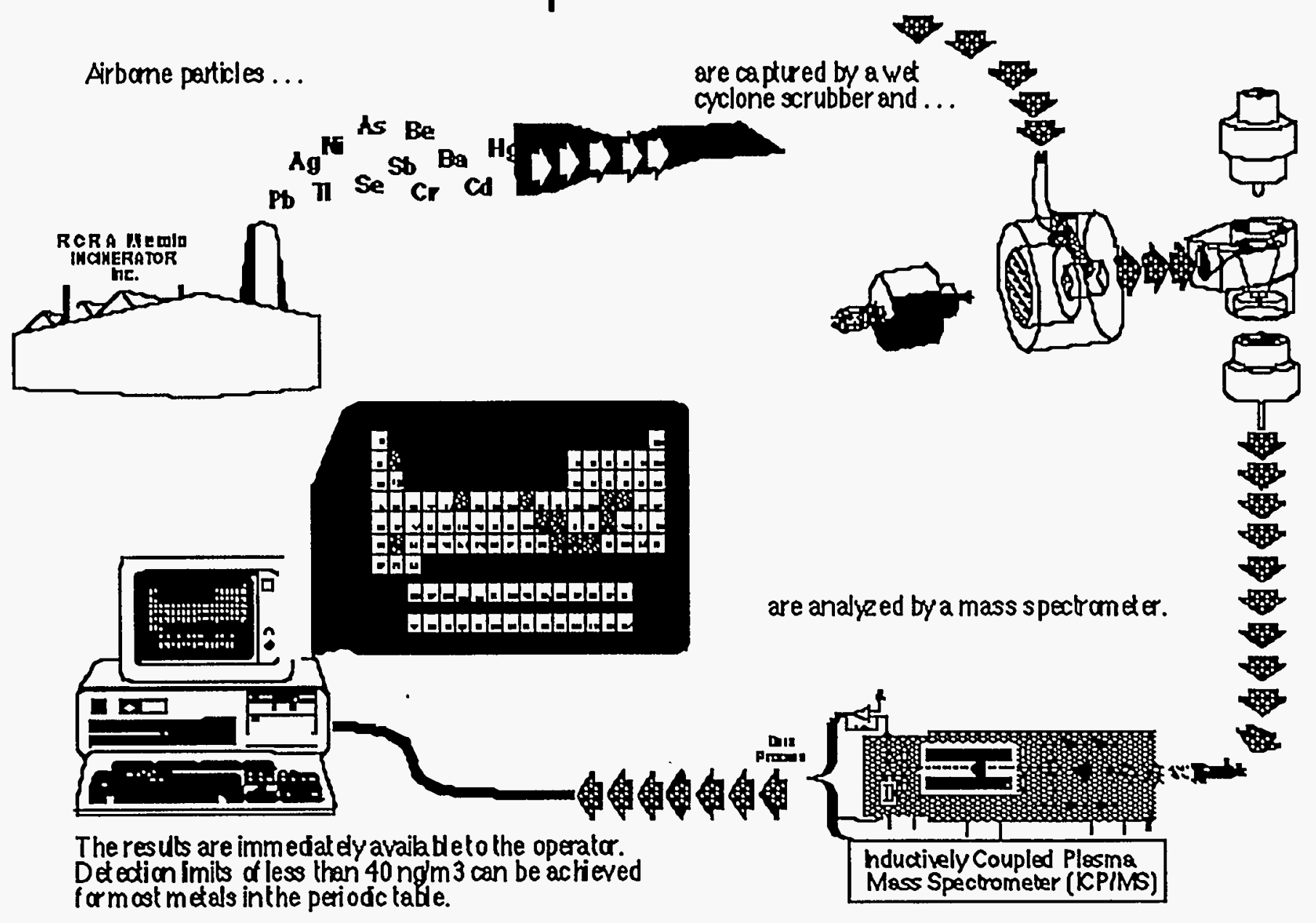




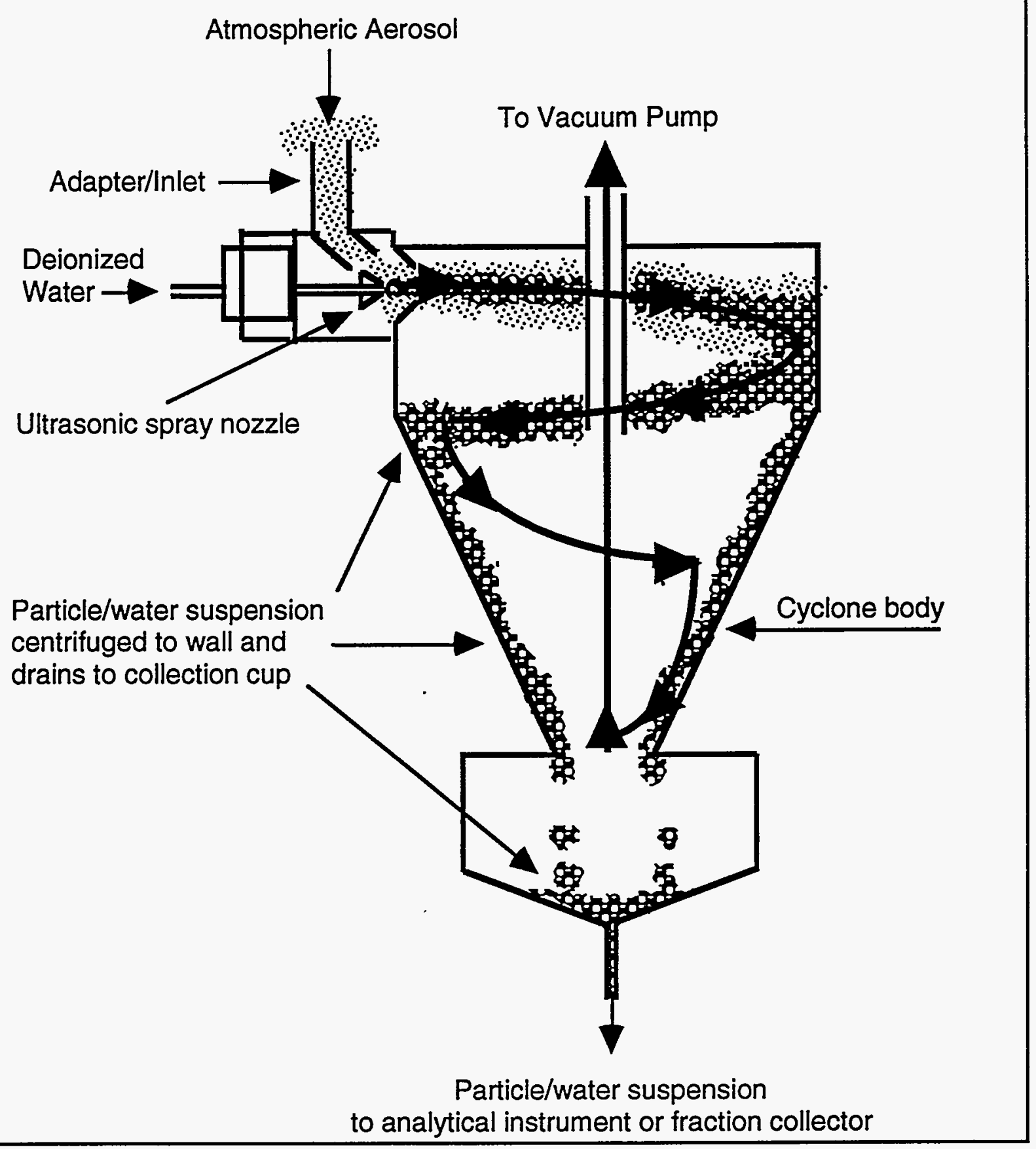

Figure 2. Schematic diagram of the Wet Cyclone Aerosol Sampler (WCAS) illustrates the operation. Atmospheric aerosol and a mist of deionized water entering the cyclone body are collected by internal aerodynamic forces. The resulting aqueous suspension is then available for immediate analysis or collection. 

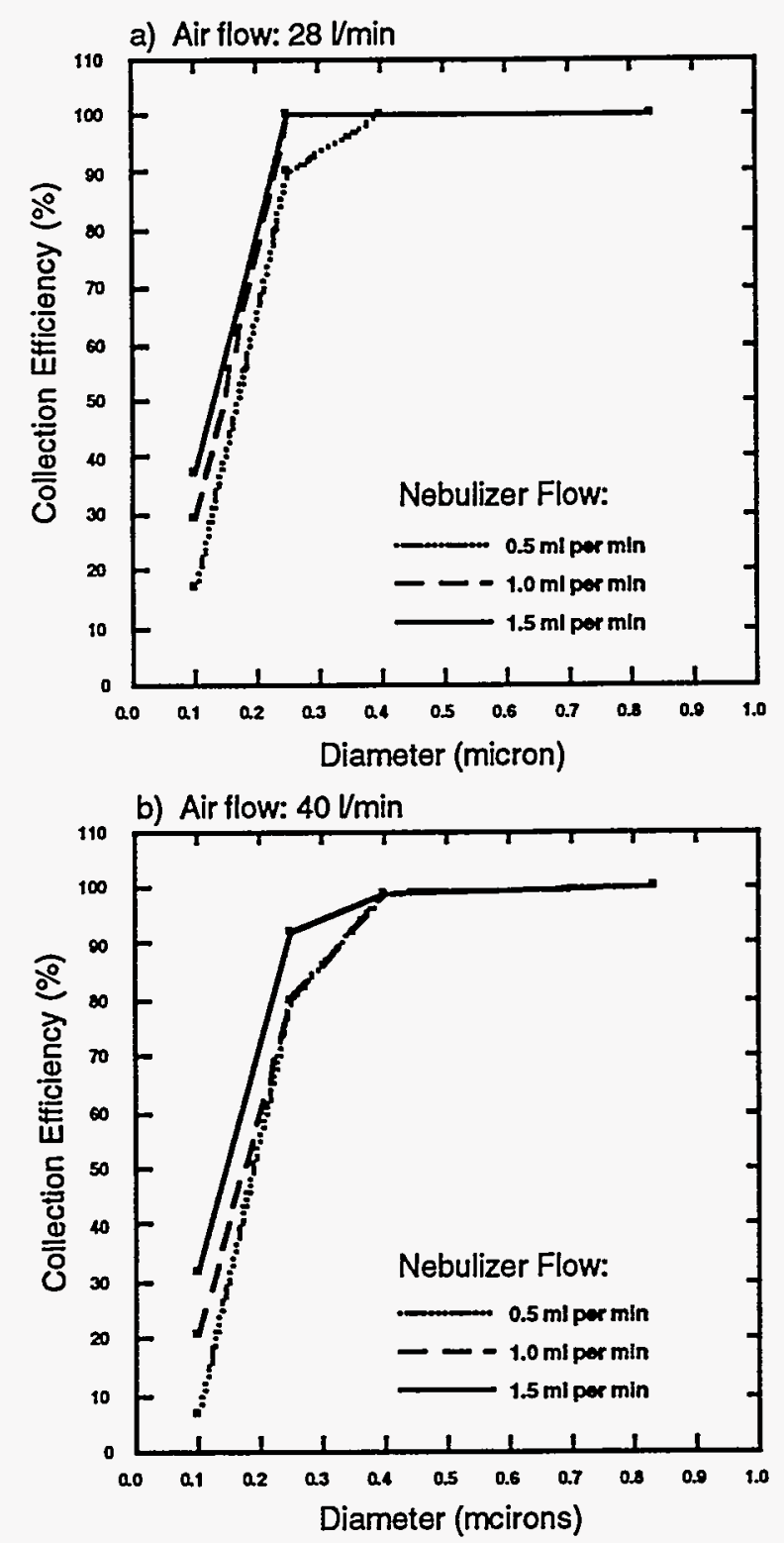

Figure 3. Collection efficiency of the Wet Cyclone Aerosol Sampler (WCAS) as a function of particle size for three different nebulizer flow rates of deionized water and two different air flow rates: a) $28 \mathrm{l} / \mathrm{min}$ and b) $40 \mathrm{l} / \mathrm{min}$. 


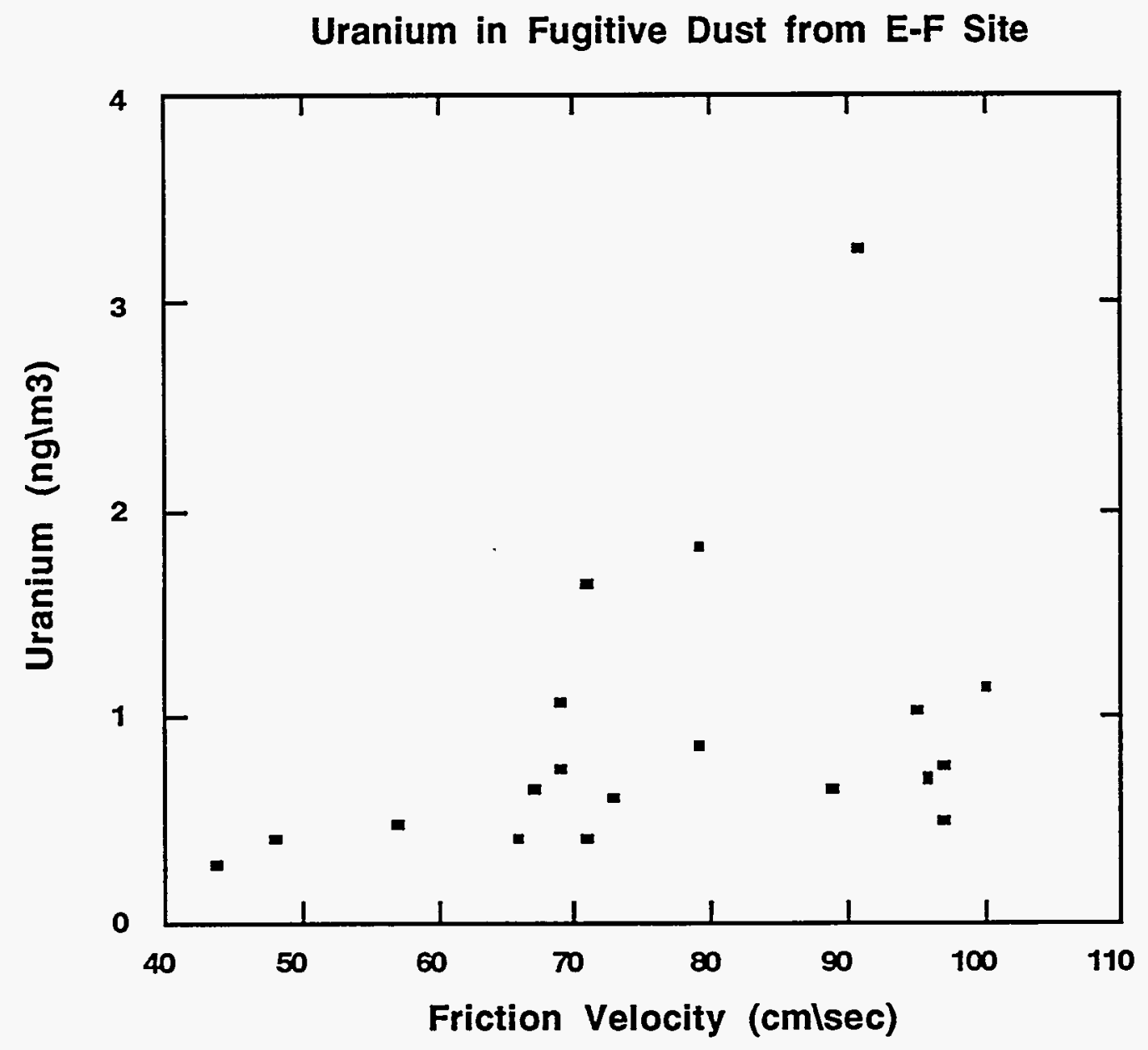

Figure 4. Uranium in fugitive dust from E-F Site as a function of friction velocity. Note the significant increase in concentration at friction velocities in excess of threshold of about 70 $\mathrm{cm} / \mathrm{sec}$. 